\title{
Energy metabolism of cancer: a suspected role of low Glutamine supply in the Warburg/Crabtree effects
}

\author{
Dell'Antone $\mathbf{P}^{*}$ \\ Dipartimento di Scienze biomediche sperimentali, Via Colombo 3, Padova, Italy
}

\begin{abstract}
For many years, now, the acridine orange dye has been successfully detecting acidity in isolated lysosomes and this has made it easy to evidence the existence of an ATP-ase responsible for the acidification of the organelles. The dye could also, as expected, detect the pH gradient between cytoplasm and organelles $(\Delta \mathrm{pHi})$ in intact cells. The detection of acidic organelles in cells can then be reasonably considered, in the absence of other specific causes of intracellular acidity deterioration, such as inhibitors of the above enzyme or $\Delta \mathrm{pHi}$ collapsing agents, as a probe qualitatively reflecting the bioenergetic status of the cells.

In the present study glutamine was tested as a feed for the oxidative phosphorylation in Ehrlich ascites tumor cells, using acridine orange just as a probe for the bioenergetic status (ATP levels) of cells. In line with a previous [1], less articulated suggestion [1], of a low involvement of suitable substrates available in cancer mitochondria at the basis of the Warburg/ Crabtree effects, the present findings support a specific role of glutamine supply in this respect. The results presented here and others taken from the scientific literature on this topic, as well as a few disposable, less conclusive in our opinion, in vivo studies, appear consistent with idea that the Warburg/Crabtree effects simply reflect the fact that the low glutamine content in cancer tissues often found in vivo, mainly supplies precursors for macromolecular synthesis rather than foraging, via a normal Krebs cycle, reducing power for the respiratory chain, and resulting in a inefficient oxidative phosphorylation pathway. Instead, a very important sustenance for oxidative phosphorylation is represented by high glutamine. Taking into account the low glutamine found in in vivo studies on cancer cells, it is argued that the Warburg effect would reflect the energy metabolism of cancer in a "milieu" with a low glutamine supply.
\end{abstract}

\section{Introduction}

Despite the complexity of the energy-providing pathways in cancer that has led recently to the concept of plasticity for cancer energy metabolism especially with regard to the role of mitochondria in providing "energy", and to that of the microenvironment of the tumor [2-5], and that there are tumors that rely on oxidative phosphorylation for their bioenergetic needs [6], the Warburg effect, or aerobic glycolysis, still remains a main hall mark for many cancer types. Indeed, it is well accepted that a lot of cancer types utilize glycolysis, rather than oxidative phosphorylation, for survival and proliferation, as also supported by the high, almost stoichiometric whit glucose, in vivo lactate production, despite oxygen availability. This is obtained by a strong increase in glucose uptake and utilization, due to an enhanced expression of glucose transporters and enzymes of the glycolytic pathway, oncogene- and mutated tumor suppressors-driven [7], well documented by the use of $18 \mathrm{~F}$ deoxyglucose uptake by cancer cells, to visualize cancer tissues, routinely in clinical settings, by positron emission tomography (PET).

While the role and pathways of glucose metabolism in cancer are well established [8,9], as providers of energy as well as precursors for biosynthesis, glutamine use by cancer cells, together with the pathways that this important fuel for cancer can take inside the cells, although largely documented [10], and emphasized with the term glutamine addiction [11], is still the subject of many investigations [12], as recent reviews well document see [13] to mention just one of them).

The ideas expressed here on how glutamine feeds oxidative phosphorylation, but only at high concentration, mainly take into account the results obtained by studying Ehrlich ascites tumor cells, as well as previous studies on cultured cells, as the few in vivo studies (see below) on this matter make it difficult, in our opinion, to derive unequivocal suggestions for what proposed here.

To asses the bioenergetic status of cell, as described below, the acridine orange dye absorbance or fluorescence was monitored [14-19] Figure 1 . The sudden absorbance change (recorded following addition of the dye to a suspension of Ehrlich ascites tumor cells or normal cells) is followed, in normal conditions (that is, in the absence of inhibitors of energy producing pathways or inhibitors of the $\mathrm{H}+$ lysosomal ATP-ase), by a slow phase of absorbance (or fluorescence) decrease (for details, see legends to Figure 1) that accompanies the dye uptake into acidic intracellular organelles as shown in Figure 1, taken from a previous study on non-proliferating rat thymocytes [19] this decrease (depending also on the cell/dye ratio), due to dimerization of the dye accumulated inside, reflects the intracellular $\mathrm{pH}$ gradient between acidic organelles and cytoplasm $(\Delta \mathrm{pHi})$. Indeed, this phase is abolished by ammonium sulfate addition, that collapses the $\mathrm{pH}$ gradient between organelles and cytoplasm, or by nigericin, as shown in Figure 1, that leads to the same result, although through an exchange between $\mathrm{H}+$ of the organelles and cytoplasmic K+ Preincubation of the cells with drugs affecting the energy-producing pathways, that is, glycolysis and

${ }^{\star}$ Correspondence to: Paolo Dell'Antone, Dipartimento di Scienze biomediche sperimentali, Via Colombo 3, Padova, Italy, E-mail: paolo.dellantone@unipd.it

Key words: aerobicglycolysis, warburg/crabtree effects, cancer oxidative phosphorylation, cancerglutamine

Received: January 23, 2021; Accepted: February 04, 2021; Published: February 18,2021 
oxidative phosphorylation, abolished the absorbance decrease phase [14] and Figure 2 symbol $\mathbf{n}$, indicating that it is the ATP pool that creates and maintains the $\mathrm{pH}$ gradient; indeed, it is a well recognised that the lysosomal $\mathrm{pH}$ gradient is created and maintained inside (lysosomes) by an ATP-driven proton pump, as demonstrated elsewhere [15-18]. Of course, inhibitors of the above enzyme, such as bafilomycin, abolished the absorbance decrease, as shown time and time again, in isolated lysosomes as well intact cells [15-18]. Thus absorbance decrease monitors the above described $\Delta \mathrm{pHi}$, albeit quantitatively, and so reflects the bioenergetic status of the cell (ATP levels).

The Ehrlich ascites tumor suspension was obtained as described [14]. The procedure how were conducted trials for $\Delta \mathrm{pHi}$ detection was the following (room temperature). Aliquots of the freshly prepared EATC suspension were submitted at the time indicated to the dye test; 18 minutes about following dye addition $(10 \mu \mathrm{M}$, final concentration), the time required for a complete dye uptake into acidic compartments, nigericin was added $(2-4 \mu \mathrm{g} / \mathrm{ml})$ and see again Figure 1, the fluorescence change recorded. What in the Figure 2 is shown is only the fluorescence change elicited by nigericin: $\Delta \mathrm{A}$ see again Figure 1 the fluorescence increase as the dye is added, and the following its decrease are not shown. As above outlined, this $\Delta \mathrm{F}$ is assumed to reflect the $\mathrm{pH}$ gradients between organelles and cytoplasm existing prior to nigericin addition and collapsed by the ionophore.

\section{Results}

Glucose inhibited, endogenous respiratory substrate driven oxidative phosphorylation low glutamine only partially restored it

In preliminar experiments, it was first analyzed oxidative phosphorylation in a glucose free medium; there was a initial, nigericin collapsed fluorescence quenching indicative that $\mathrm{pHi}$ gradients pre- existed, presumably in part due to endogenous respiratory substrates [14,20] (Figure 2). Indeed oligomycin, inhibitor of oxidative

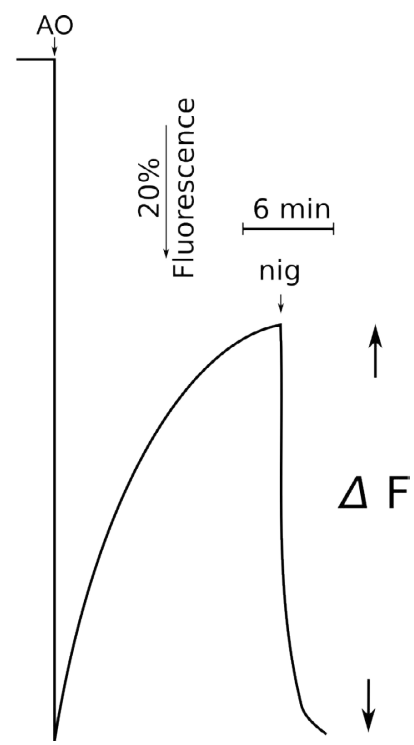

Figure 1. How to detect intracellular $\mathrm{pH}$ gradients $(\Delta \mathrm{pHi})$ measuring $\mathrm{AO}$ absorbance or fluorescence change: a dual wavelength spectrophotometer can be used, employing 492 $\mathrm{nm}$ as measuring and $550 \mathrm{~nm}$ as reference wavelength; or fluorimetrically, by using thus as excitation $492 \mathrm{~nm}$ and emission $550 \mathrm{~nm}$ wavelength. The figure refers to an experiment conducted in normal rat thymocytes; nigericin addition $2-4 \mu \mathrm{g} / \mathrm{ml}$. Other details as to cell preparation and suspending medium as in (19)
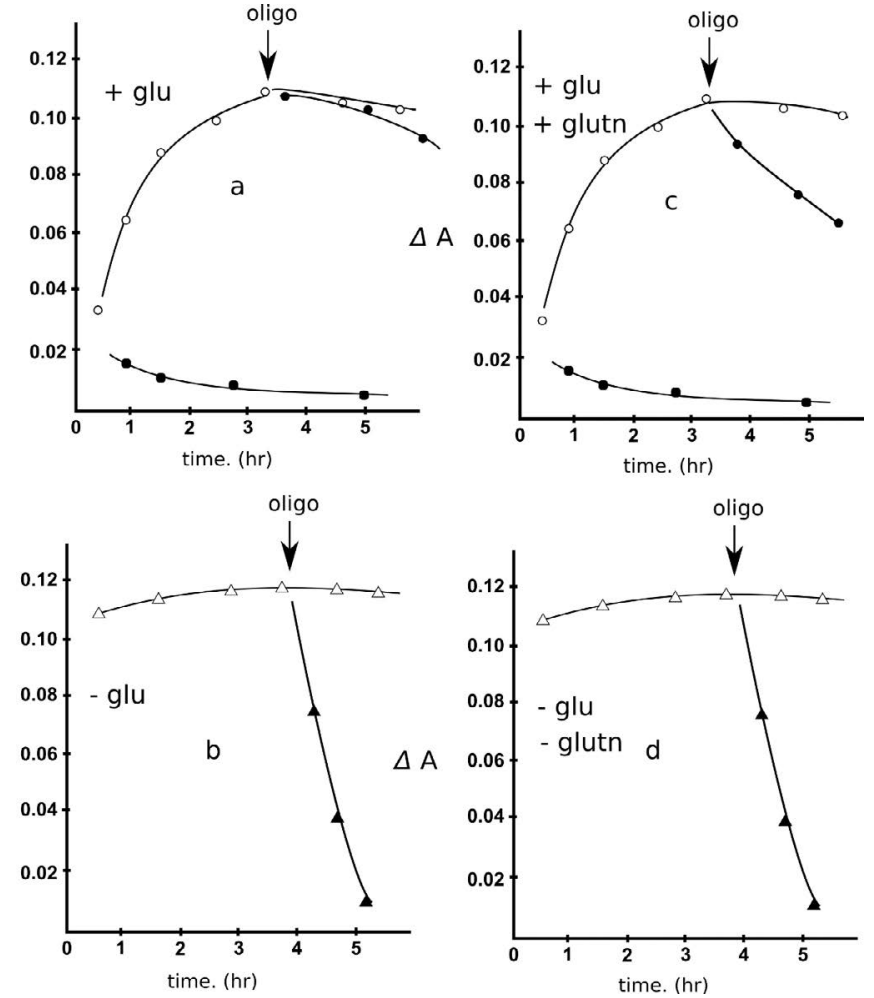

Figure 2. Glutamine supports oxidative phosphorylation in Ehrlich ascites tumor cells. Inhibitory role of glucose at low or not glutamine supply. The procedure for the ascertainment of glutamine-driven oxidative phosphorylation activity in different conditions that is, in the presence or absence of glucose $(8 \mathrm{mM})$ is described in the text and rests on the inhibitory role of oligomycin $(1.5 \mu \mathrm{g} / \mathrm{ml})$ on oxidative phosphorylation. AO concentration $10 \mu \mathrm{M}$, cell concentration, EATC suspending medium , nigericin addition $2-3 \mu \mathrm{g} / \mathrm{ml}$. and other specifications as in reference (14); aliquots of the cell suspensions were supplemented or not with glucose $8 \mathrm{mM}$ or glutamine $1 \mathrm{mM}$, or both, when indicated. When indicated (Fig 2 a,c $\mathbf{m}$ ), oligomycin $2 \mu \mathrm{g} / \mathrm{ml}$ and deoxyglucose $5 \mathrm{mM}$ were present.

Abbreviations: Glu: Glucose; Gltn: Glutamine; Oligo: Oligomycin

phosphorylation, reversed totally the pHi gradients driven fluorescence change (Figure $2 \mathrm{~b}$ ) in a time depending required to consume the cellular ATP. However in some case the initial fluorescence was reduced in extent. This point was not further investigated neither clarified, but of course it reflected a different initial ATP content in different preparations.

In a glucose medium, it was observed soon a initial decrease in ATP levels, really variable in extent in different preparations (Figure 2), likely due to ATP consumption in the first steps of glycolysis), but restored in some time ("long" at room temperature), that has been described in more studies [14] references therein likely due to restoration by glycolysis of an ATP pool. Indeed the presence of glucose made oligomycin (inhibitor of oxidative phosphorylation) ineffective to collapse this $\Delta$ pHi gradients, endogenous fuels supported [20], indicating low, if any, oxidative phosphorylation participation to the creation of the $\mathrm{pH}$ gradients when glucose is present (Crabtree effect).

Again in the presence of glucose, low glutamine $(1 \mathrm{mM})$ and eventual endogenous respiratory substrates [20], as Figure 2 shows, restored some oxidative phosphorylation, detected, as usual by a (partial now) oligomycin effect indeed not a complete pHi gradient collapse due to deterioration of $\mathrm{pH}$ gradients was observed, as instead seen in the absence of glucose Figure 2. 
However, by the present data it was difficult to quantify the effect of glutamine (tht could be major than that observed, for the presence of residual endogenous respiratory substrates and for $\mathrm{pHi}$ gradients could also be due to glycolytic ATP. Thus this point would deserve further investigation.

Neither less, too sum up: despite the presence of glucose that should inhibit oxidative phosphorylation, (see above) glutamine concentration as low as $1 \mathrm{mM}$, partially restored it (no complete Crabtree effect), a finding revealed as usual by oligomycin (arrows) elicited some $\mathrm{pHi}$ gradients deterioration. Instead in the absence of glucose oxidative phosphorylation was completely active ( $\mathrm{pHi}$ gradients created by glutamine driven oxidative phosphorylation).

It is reasonable to think that high glutamine ( $4 \mathrm{mM})$ usually employed in studies in cancer cells in culture, should support oxidative phosphorylation also in glucose medium, as reported in more studies, as throughly discussed below in a tentative scheme to rationalize the above and other findings.

\section{Discussion}

\section{Glutamine and Crabtree effect: only at low glutamine?}

Freshly prepared Ehrlich ascites tumor cells exhibited oxidative phosphorylation, since oligomycin inhibited the ATP-driven dye uptake, too in a glucose and glutamine free medium (Figure 2), thus supported, it is like, by endogenous or residual feeds producing oxidizable substrates (NADH etc) in the suspending medium [20] preincubation with glucose totally inhibited oxidative phosphorylation in our trials, as demonstrated by the lack of effect of oligomycin on ATP levels (Crabtree effect), Figure 2. So, in this condition ATP levels were maintained by glycolysis alone. (Of course oligomycin plus deoxyglucose totally prevent the establishment of full ATP levels ref 14).

A similar finding, demonstrating the Crabtree effect, as glucose inhibition of respiration ( $70 \%$ inhibition of glutamine, or other oxidaizable substrates, supported, respiration rate) was reported in the same cell type, again in the presence of glucose and low $(0,5 \mathrm{mM})$ glutamine [21] (Table 1).

Moreover in a study always on utilization of energy substrates for Ehrlich ascites tumor cells, (with glucose $5 \mathrm{mM}$ and glutamine $0,5 \mathrm{mM}$ again), consistent with the idea that it is glucose that activates proliferation [22], while the rate of glutamine oxidation was significantly decreased (by 70\%)in the same condition as above reported [21] glutamine consumption was increased (in the presence of glucose) in respect to glutamine alone [23] this supports the idea that (low) glutamine was used more as precursors provider than to feed the respiratory chain, as will be discussed below.

Instead in another study it was reported (in EATC) that glucose did not inhibits glutamine driven respiration respiration but instead activated it, but a higher $(2 \mathrm{mM})$ glutamine concentration was used, thus supporting what above hypothesized. In our findings: in a glucose free medium glutamine, at slightly higher levels $(1 \mathrm{mM})$ than those measured in tumors (see below), but higher than that used in the above cited studies, restored a full oxidative phosphorylation activity, again revealed by the effect (reduction) of oligomycin on ATP levels (Figure 2) monitored as pHi gradients set up), but, and presumably at higher concentrations (to be verify) also in a glucose medium.
Thus the absence of glucose that should drive the proliferatve pathways, endogenous metabolytes migth be involved only in provide reducing power for oxidative phosphorytylation. Instead in a glucose medium, gutamine caused only a partial reduction in $\Delta$ phi, ssuggesting its utilization in other pathwys, presumably involved in setting up the porliferative process. Moreover in a study on Ehrlich ascites tumor cells. To sum up the above findings seems again support the view that, at low glutamine supply, mitohcondrial metabolites are more involved in other pathways rather than in activating respiration, presumably to provide precursors for biosynthesis at higher also in supporting the respiration pathway. This point will be further explored below.

\section{Glutamine and oxidative phosphorylation in cancer}

That cancer cells may accomplish oxidative phosphorylation is well documented, (it certainly is for Ehrlich ascites tumor cells and for this study, as well as for different cells lines in culture or homogenates of hepatomas $[6,25,26]$ but often, to the best of our knowledge, in the presence of glutamine at high concentration of 4-5 $\mathrm{mM}$, as it usually happens with cultured cells [27-29] a comparison between ATP production in the two pathways (glycolysis vs oxidative phosphorylation), however, was not provided, at least in the above mentioned studies [27-29].

So, in order to observe a robust oxidative phosphorylation, high (mMolar) glutamine was required [26-29] and this occurs only in cultured or isolated cells (but see ref 6 for some apparent discrepancy from this statement).

The hypothesis of this study and of my previous study [1] is that the Crabtree effect in cancer cells, when present, is not a phenotype acquired by cells progressing toward a malignant transformation, but, rather, appears in concurrence with a shortage of proper metabolites, having cancer cells at disposal $[30,31]$ due to a dysregulated Krebs cycle, to support oxidative phosphorylation. The role of substrate availability in mitochondrial respiration rate has been reported in the above mentioned study [30], in which galactose substituting glucose enhanced the rate, but glutamine $4 \mathrm{mM}$ together with galactose was added!

For, in order to satisfy cancer growth and proliferation, mitochondrial metabolites should be principally in the present view, at low or no glutamine added, employed more than to feed the respiratory chain, to provide other pathways, partly suggested in a previous study [1] such as synthesis of amino acids, production of NADPH via the malic enzyme [29,32]. for the synthesis of lipids following the export of malate into the cytoplasm, thus resulting in an altered malate/aspartate shuttle that instead would provide NADH to be translocated into the mitochondria.

It is believed that this pathway (via malic enzyme) produces more NADPH than the penthose phosphate pathway [32]. In a study on the Crabtree effect in proliferating lymphocytes, the involvement of defective reducing power translocation into mitochondria, due to impairment of the malate- aspartate shuttle was considered [20]. As elsewhere proposed [1], other pathways could be involved to lower the reducing power supply in mitochondria; for instance, cytoplasmic glycerol phosphate could be directed again to lipids synthesis, as hypothesized [1], instead of providing or the respiratory chain. And in a study on pancreatic cancer cells a metabolic pathway other than proposed in [32] a Kras-regulated metabolic pathways was proposed, 
leading again to malate being converted to pyruvate, via mitochondrial transaminase with glutammate, to generate again, finally, NADPH [33].

Again, a truncated Krebs cycle that would use citrate, once extruded from mitochondria [34] again for lipids synthesis [35] could take away reducing power. And so could takeaway reducing power the reductive carboxylation of alpha ketoglutarate inside the mitochondria [35-37], that would again consume reducing power resulting from glutamate dehydrogenase) to produce citrate to be exported again into the cytoplasm for lipid synthesis. Of course, the machinery that leads to lipids synthesis must be well active and operating in more pathways, if a cancer cell is to give origin to a daughter cell. Notice than more of these pathways use glutamine as a source.

Thus, to synthesize, does aerobic glycolysis, generally observed in cancer in vivo, simply reflect that glucose-fed and thus proliferating cancer cells, require precursors for macromolecule biosynthesis and this exhausts the low content of endogenous glutamine or is glutamine that cancer cells can obtain normally by the environment in vivo just consumed in not reducing power producing pathways, so that oxidative phosphorylation takes place only in the presence of high glutamine contents such as those available in cultures. Of course, cancer cells, in order to meet the requirements of growth and proliferation, prefer, at low glutamine, to pursue precursor supply instead of ATP production that can be well supported by glycolysis. Some studies $[29,33]$ and supported by theoretical considerations [34] claimed that glutamine's main role in cancer is to supply lipids synthesis. At high glutamine levels, such as those available in cultured cells when the precursors supply is "saturated ", glutamine can be provided to reinforce the Krebs cycle.

In conclusion in our view: in cancer cells, in the presence of glucose, mitochondria are more engaged in supplying precursors for macromolecule synthesis than in providing reducing power for the respiratory chain, when the support of glutamine is scarce. If the values for glutamine concentration found in vivo could be reinforced by new determinations and so firmly established, low or no glutaminedriven oxidative phosphorylation should occur; this would lead to the activation of glycolysis, in the opposite direction to the normal Pasteur effect, that is to say, to the Warburg effect.

It is currently thought that the Pasteur effect (oxygen available -low glycolysis) is not operative in cancer (aerobic glycolysis) however, it could not be true, since aerobic glycolysis might be simply due to low glutamine levels in cancer cells that would not allow observable oxidative phosphorylation, because of the poor availability of suitable substrates as a consequence, as amply above discussed, of the proliferative process in progress. Of course, having oxygen available is a necessary but not sufficient condition for oxidative phosphorylation to take place.

\section{To explore glucose oxidation in cancer (also in vivo)}

Another crucial question of course is if glucose is oxidized in vivo in tumor cells to sustain oxidative phosphorylation, and if so, to what extent.

To this purpose studies have been performed using NMR spectroscopy, after infusing nutrients labelled with $13 \mathrm{C}$ into animals bearing human glioblastoma cells the tumor was dissected from the brain and specimens were analyzed for metabolites that showed high lactate production, as expected (a robust aerobic glycolysis), but also a contribute of glucose to oxidative phosphorylation as revealed by the presence of marked (13 C) metabolites feeding the TCA cycle [36-38].
Another study [39] conducted on tumor samples removed from a human glioblastoma confirmed that there us some contribution of glucose oxidation to the energy metabolism of cancer cells. However, it is possible that, as in the previously cited study, when specimens were extracted from the tumor and analyzed to ascertain the possible involvement of glucose-derived metabolites in oxidative phosphorylation, the presence of the TCA cycle (deduced by the above spectroscopic analysis) might be due to lactate (marked) deriving from the environment of the tumor; likely withdrawn together with the tumor specimens and used in vivo to feed some other, oxygenated, tumor cells [2].

Another study using glioblastoma cells in culture [29] investigated a possible role of glucose oxidation using NMR spectroscopy in the absence of glutamine, however, the consumption of glucose was found to result prevalently in acid lactate production then supporting a high level of aerobic glycolysis as usual in cancer.

Finally, in the same study, but in the presence of glutamine," the TCA cycle was active, but it was characterized by an efflux of substrates for use in in biosynthetic pathway, especially fattycid synthesis. However, too in this study, as in others, no oxidative phosphorylation activity was measured, which would have been useful in order to compare the contribution of each pathway to the energy status of these cancer cells. Thus, the detection of marked metabolites of Krebs cycle (dysregulated) does not necessarily imply their massive involvement in oxidative phosphorylation. Once again, to obtain a significant picture of the question, the contribution of glycolysis versus oxidative phosphorylation should be quantified in the above studies, as further discussed below.

\section{Oxidative phosphorylation against glycolysis}

In vivo, aerobic glycolysis, which is considered the main hallmark of tumors, is well documented by the use in of 18F- Positron emitting tomography with $18 \mathrm{~F}$ deoxy glucose administration. But in a muchappreciated study [25], ZU and Guppy report the results of additional instances in which both glycolysis (lactate production) and oxidative phosphorylation (P/O ratio) were measured, in cultured or Ehrlich ascites tumor cells or homogenates from rat hepatomas. It was reported that both, although at different degrees, consistently contribute to ATP production. Thus it was concluded that tumors do not strictly observe aerobic glycolysis it was argued that $18 \mathrm{~F}-\mathrm{PET}$ only registers massive glucose uptakes that could in part be redirected to other pathways (such as the penthouse phosphate pathway, serine synthesis, lipid biosynthesis via glycerol phosphate and others). It was argued that glucose might take pathways not conducing specifically to glycolysis even though other studies demonstrated that the amount of glucose utilized in the above pathways would be but a small part of the total glucose consumed (10\%) moreover, this consideration contrasts with the massive lactate production by cancer cells recorded also in vivo. As above specified, studies cited by Guppy et al. were conducted on cultured cells or Ehrlich ascites tumor cells but more often in homogenates of rat hepatomas; in all cases, glutamine was given (0.5- $4 \mathrm{mM}$ as reported in $\mathrm{Zu}$ and Guppy study) as usual and of course with easy access to the cells or enzymes; however, for tumor homogenates the determination of oxidative phosphorylation might give raise to some perplexity, considering that some activities could require intact structures. Of course, it would be very important to ascertain if, when low glutamine was supplied, this resulted in lower oxidative phosphorylation and a corresponding increase in glycolytic ATP. Finally, it has to be mentioned that some authors hypothesize that glutamine might have an important role in inducing oxidative phosphorylation (as well as proliferation) only in cells in culture [40]. 


\section{Glutamine in vivo and aerobic glycolysis}

A crucial point to give support to the above suggestion as to the role of glutamine (mMolar) in eliciting oxidative phosphorylation in vivo or not, as observed in cultures, (but at mMolar concentrations) is, of course, glutamine concentration in cancer in vivo to be compared with normal tissues $[6,7,8,37]$. To the best of this author's knowledge, there are not many in vivo tumor studies that focus on this, and the existing ones report normally for plasma and muscle less of 1 mmolar and more mMolar respectively. However, some report that, at difference of normal tissues or healthy tissues in the vicinity of the cancer area, the concentration in cancer tissues was found many fold lower, that is about $0.5 \mathrm{Mm}$ or lower [41]. Moreover, it is suspected that no more than 0.5 $\mathrm{mM}$ of glutamine is taken up by cancer cells [42]. Thus, one may wonder if massive oxidative phosphorylation, such low glutamine-driven one, could exist (as in cultured or Ehrlich ascites cells at high glutamine) or if aerobic glycolysis predominates in vivo as argued by $18 \mathrm{~F} \mathrm{PET}$ and the observed massive lactate production. Moreover few in vivo studies, if any, report both glycolysis and oxidative phosphorylation together as more times pointed out in this study.

Finally, because of the multiple functions that glutamine can perform inside cancer cells, it is reasonable to think that the rate limiting step of the entire pathway is glutamine sequestration by cancer cells. So the glutamine concentration found [42] might be much lower inside than the value in the plasma or in other normal tissues [40], even though some hypothesize that this is due just to the high glutamine consumption [41]. In any case, such a low glutamine value cannot support high oxidative phosphorylaton in vivo to consider results with EATC.

\section{A pyruvate -malate cycle in tumors?}

A further way by which the mitochondrial matrix could became reducing power-deficient? Old studies reported no activation of pyruvate carboxylase [29] at least in some cancer types; pyruvate carboxylase normally reinforces the Krebs cycle, together with glutamine, but enters also in other mitochondrial reactions, such as transaminase with glutamate and many others again. Recent studies conducted in vivo suggest a completely different picture for this enzyme: its strong activation at least in some tumors [43-46]. Oxoloacetate produced inside mitochondria by the above enzyme can feed the Krebs cycle, provided that the pyruvate dehydrogenase kinase that inhibit pyruvate dehydrogenase is not over-expressed. Provided that this do not occurs, then oxaloacetate could take the opposite direction from that of the usual of the Krebs cycle (given the low acetylcoenzyme A) and together with malate dehydrogenase being strongly directed toward malate synthesis [47], could generate malate; once extruded from mitochondria it could generate NADPH via malic enzyme, to feed the lipids synthesis, and restore pyruvate, so realizing a hypothetical pyruvate cycling. Should this hypothesized pathways really exist in tumors, a lot of reducing power would be again lost for mitochondria.

\section{What is the role of glucose in sustaining the Crabtree effect?}

It is well accepted, generally, that glucose is essential for cell proliferation [22] in which it plays the role of master metabolite in cancer proliferation, despite a few studies $[28,48]$ claiming that glutamine can substitute glucose, also in eliciting proliferation. However, this does not sound valid, considering the role of the glycolysis intermediates in providing precursors for biosynthesis. In the above-mentioned study on proliferating lymphocytes [22], the consensus was that glucose induces a transition that leads to aerobic glycolysis and proliferation. High glucose consume is required only in order to provide precursors for macromolecule synthesis $[8,9,49]$ or does glucose, made available in high amount as that taken up under the expression of oncogenes act as a "trigger" as in yeasts, of the proliferative process? In this case, it could have a role in the interplay with signal transduction pathway.

Thus, how does glucose relate with oncogenes and tumor suppressors? As, a glucose-to-genes link? Glucose as hormone? [5053]. This is an intriguing topic, which, again, is neither well understood nor thoroughly explored that should give further insight in the comprehension of cancer genesis.

\section{Conclusion and perspectives}

The aim of the present study is to attempt an explanation of the Warburg/ Crabtree effect that occurs in cancer cell in vivo, taking into account, as reliable, available data on the low glutamine amount found in vivo in cancer and suggesting a main role of glutamine at low concentrations mainly in providing precursors for macromolecule synthesis rather than in feeding reducing power to the respiratory chain via the Krebs cycle: therefrom the Crabtree effect. At high concentrations, instead, glutamine could also play the role of feeding the Krebs cycle to produce reducing power, as is already well documented, but in isolated or in cultured cells.

As for the role of glucose in sustain oxidative phosphorylation, existing in vivo studies did not give, in our opinion, clear answers in that the data obtained did not provide unequivocal interpretation. Whether glutamine supports both oxidative phosphorylation as well as proliferation in cells in culture, thus replacing the role of glucose, appears rather difficult to be ascertaine the considering the fundamental role of glucose in supplying precursors for biosynthesis.

Since in vivo studies often reverse the results obtained with the help of cultured cells, the following are necessary: i) A thorough study comparing the activity of key enzymes involved in the energy metabolism (such as pyruvate dehydrogenase /pyruvate kinase pyruvate carboxylase and other Krebs cycle) ii) the comparison differentiating between cultured cells (ex vivo), transformed cells in culture with oncogenes, in vivo cancer cells xenografts, and samples drown from cancer tissues of patients, to be compared with neighboring healthy tissues; again further in vivo studies with $\mathrm{C}$ or $\mathrm{N}$ isotopes by means NMR spectroscopy.

Figure 1 How to detect intracellular $\mathrm{pH}$ gradients $(\Delta \mathrm{pHi})$ measuring AO absorbance or fluorescence change, a dual wavelength spectrophotometer can be used, employing $492 \mathrm{~nm}$ as measuring and $550 \mathrm{~nm}$ as reference wavelength or fluorimetrically Figure 2, by using as excitation $492 \mathrm{~nm}$ and emission $550 \mathrm{~nm}$ wavelength. The figure refers to an experiment conducted in normal rat thymocytes nigericin addition $2-4 \mu \mathrm{g} / \mathrm{ml}$. Other details as to cell preparation and suspending medium as in [19]. Figure 2 Glutamine supports oxidative phosphorylation in Ehrlich ascites tumor cells. Inhibitory role of glucose. at low or not glutamine supply.

The procedure for the ascertainment of glutamine-driven oxidative phosphorylation activity in different conditions that is, in the presence or absence of glucose $(8 \mathrm{mM})$ is described in the text and rests on the inhibitory role of oligomycin $(1.5 \mu \mathrm{g} / \mathrm{ml})$ on oxidative phosphorylation. AO concentration $10 \mu \mathrm{M}$, cell concentration, EATC suspending medium, nigericin addition $2-3 \mu \mathrm{g} / \mathrm{ml}$. and other specifications as in 
reference (14); aliquots of the cell suspensions were supplemented or not with glucose $8 \mathrm{mM}$ or glutamine $1 \mathrm{mM}$, or both, when indicated. When indicated (Figure 2), oligomycin $2 \mu \mathrm{g} / \mathrm{ml}$ and deoxyglucose 5 $\mathrm{mM}$ were present.

\section{Conflict of interest}

None.

\section{References}

1. Dell'Antone P (2012) Energy metabolism in cancer cells: How to explain the Warburg and Crabtree effects? Medical Hypotheses 79: 388-392. [Crossref]

2. Feron O (2009) Pyruvate in lactate and back: From the Warburg effect to symbiotic energy fuel exchange in cancer cells. Radiotherapy and Oncology 92: 329-333.

3. Nakajiam EC, Van Houten BV (2013) Metabolic symbiosis in cancer: Refocusing the Warburg lens. Mol Carcinog 52: 329-337.

4. Ocana MC, Martinez-Poveda B, Quesada AR, Medina MA (2018) Metabolism within tumor microenvironment and its implication on cancer progression. An ongoing therapeutic target. Medicinal Research Review 39.

5. Zheng J (2012) Energy metabolism of cancer: Glycolysis versus oxidative phosphorylation. Oncol Lett 4: 1151-1157.

6. Chen JQ, Russo J (2012) Dysregulation of glucose transport, glyoclysis, tca cycle and glutaminolysis by oncogenes, tumor suppressors in cancer cells. Biochem Biophys Acta 1826: 370-384. [Crossref]

7. Lunt SY, Vander Heiden MG (2011) Aerobic glycolysis: meeting the metabolic requirements of cell proliferation. Annu Rev Cell Dev Biol 27: 441-464.

8. Vander Heiden MG, Cantley LC, Thompson CB (2009) Understanding the Warburg effect: the metabolic requirements of cell proliferation. Science 324: 1029-1033.

9. Yang L, Venneti S, Nagrath D (2017) Glutaminolysis: A hallmark of cancer metabolism Annu Rev Biomed Eng 19: 163-194.

10. Wise DR, Thompson CB (2010) Glutamine addiction: A new therapeutic target in cancer. Trends Biochem Sci 35: 427-433.

11. Cluntun AM, Lukey MJ, Cerion RA, Locasade JW (2017) Glutamine metabolism in cancer: understanding the heteogeneity. Trends Cancer 3: 169-180.

12. Bott AJ, Maimouni S, Zong WX (2019) The pleiotropic effect of glutamie utilization in cancer. Cancer (Basel) 11: 770 .

13. Dell'Antone P (1994) Metabolic pathways in Ehrlich ascites tumor cells recovering from a low bioenergetic status. FEBS Letters 350: 183-186.

14. Dell'Antone P (1979) Evidence for an ATP- driven proton pump in rat liver lysosomes by basic dyes uptake. Biochem Biophys Res Commun 86: 180-189.

15. Moriyama Y, Takano T, Ohkuma S (1982) Acridine orange as a fluorescence probe for lysosomal proton pump. The Journal of Biochemistry 92: 1333-1336.

16. Altan N, Chen Y, Schindler M, Simon M (1999) Tamoxifen inhibits acidification in cells independent of the estrogen receptor. Proc Natl Acad Sci USA 96: 4432-4437.

17. Palmgren MG (1991) Acridine orange as a probe for measuring ph gradient across membranes. mechanism and limitations. Analytical Biochemistry 192: 316-321.

18. Dell'Antone $\mathrm{P}$ (2006) Inactivation of $\mathrm{H}+$-vacuolar ATP-ase by the Energy Blocker 3-Bromopyruvate, a New Antitumor Agent. Life Sci 79: 2049-2055.

19. Guppy M, Greiner E, Brandt K (1993) The role of the Crabtree effect and an endogenous fuel in the energy metabolism of resting and proliferating thymocytes. Eur J Biochem 212: 95-99.

20. Medina MA, Sanchez-Jimenez F, Marquez FJ (1988) Glutamine and glucose as energy substrates for Ehrlich ascites tumour cells. Biochemistry International 16: 339-347.

21. Greiner EF, Guppy M, Brand K (1994) Glucose is essential for proliferation and the glycolytic energy induction that provokes a transition to glycolytic energy production. The Journal of Biological Chemistry 269: 31484-31490.

22. Segura JA, Medina MA, Alonso FJ (1989) Glycolysis and glutaminolysis in perifused Ehrlich ascites tumor cells. Cell Biochemystry and function 7: 7-10.

23. Kovacevic, Morris HP (1972) The role of glutamine in the oxidative metabolism of malignant cells. Cancer Res 32: 326-333.
24. X1 Z, Guppy M (2004) Cancer metabolism: fact, fantasy, and fictions. Biochem Biophys Res Res Commun 313: 459-465.

25. Elwood JC, Lin YC, Cristofolo WJ, Weinhouse S, Morris HP (1963) Glucose utilization in homogenates of the Morris Hepatoma 5123 and related tumors. Cancer Research 23: 906-913. [Crossref]

26. Fan J, Kamphorst JJ, Mathew R (2013) Glutamine- driven oxidative phosphorylation is a major ATP source in transformed mammalian cells in both normoxia and hypoxia. Molecular System Biology 9: 712.

27. Le A, Lane AN, Hamaker M (2012) Glucose-independent glutamine metabolism via TCA cycling for proliferation and survival in B-cells. Cell Metab 15: 110-121.

28. De Berardinis RJ, Mancuso A, Daikhin E (2007) Beyond aerobic glycolysis: transformed cells can engage in glutamine metabolism that exceeds the requirement for protein and nucleotide synthesis. Proc Natl Acad Sci USA 104: 19345- 19350.

29. Rossignol R, Gilkerson R, Gilkerson G, Aggeier R (2004) Energy substrate modulates mitochondrial structure and oxidative capacity in cancer cells. Cancer Res 64: 985-993.

30. Zhdanov AV, Waters AHC, Golubeva AV (2014) Availability of the Key metabolic substrates dictates the respiratory response of cancer cells to the mitochondrial uncoupling. Biochim Biophys Acta 1837: 51-62.

31. De Berardinis RJ, Sayed N, Ditsworth D, Thompson CB (2008) Brick by brick: metabolism and tumor cell growth. Current Opinion in Genetics \& Development 18 54-61.

32. Son J, Lyssiotis CA, Ying H (2013) Glutamine supports pancreatic cancer growth through a KRAS- regulated metabolic pathway. Nature 496: 201-205.

33. Scott DA, Richardson AD, Fipipp FV (2011) Comparative metabolic flux profiling of melanoma cell lines. Beyond the Warburg effect. The Journal of Biological Chemistry 286: 42626-42634.

34. Parlo RA, Coleman RS (1984) Enhanced rate of citrate export from cholesterol rich hepatoma mitochondria. The truncated Krebs cycle and other metabolic ramifications of mitochondrial membrane cholesterol. J Biol Chem 259: 9997-10003.

35. Gameiro PA, Bell El, Mattaini KR (2011) Reductive glutamine metabolism by IDH1 mediates lipogenesis under hypoxia. Nature 481: 380-384

36. Altman BJ (2016) From Krebs to clinic: glutamine metabolism to cancer therapy. $\mathrm{Nat}$ Rev Cancer 16: 619-634.

37. Marin-Valencia I, Yang C, Mashimo T (2012) Analysis of tumor metabolism reveal mitochondrial glucose oxidation in genetically diverse, human glioblastomas in the mouse brain in vivo. Cell Metab 15: 827-837.

38. Maher EA, Marin-Valencia I, Bachoo RM (2012) Metabolism of [U-13C] glucose in human brain tumor in vivo. NMR Biomed 25: 1234-1244.

39. Christopher TH, Wasti AT, DeBerardinis RJ (2013) Glutamine and cancer: cell biology, physiology, and clinical opportunity. J Clin Invest 123: 3678 -3689.

40. Jiang J, Srivastava S, Zhang JI (2019) Starve cancer cells of glutamine: Break the spel or make a hungry monster? Cancers (Basel) 11: 804-820. [Crossref]

41. Kallinovski F, Runkel S, Fortmeyer HP (1987) L- glutamine: A major Substrate for Tumor cells in vivo ? J cancer Res Clin Oncol 113: 209-215.

42. Christopher TH, DeBerardinis RJ (2015) In vitro analysis of lung cancer metabolism: nothing as the real think. $J$ Clin Invest 15: 495-497.

43. Wilmanski TM, Shinde AS, Donkin SS (2018) Pyruvate carboxylase is essential for breast cancer metastasis in vivo FASEB. Journal Biochemistry and Molecular Biology 12.

44. Cheng T, Sudderth J, Yang C (2011) Pyruvate carboxylase is required for glutamine01independent growth of tumor cells. PNAS 4108: 8674-8679.

45. Sellers K, Fox MP, Bousamra M (2015) Pyruvate carboxylase is critical for non-smallcell lung cancer proliferation. J Clin Invest 125: 687-686.

46. David LN, Michael Cox (1982) Lehninger Principi di biochimica. Ed. $7^{\text {th }}$ page 427.

47. Chen J, Lee HJ, Wu X (2015) Gain of glucose-independent growth upon metastasis of breast cancer cells to the brain. Cancer Res 75: 554-565. [Crossref]

48. Sandulake VC, Ow TJ, Pikering CR (2001) Glucose, not glutamine, is the dominan energy source required for proliferation and survival of head and neck squamous carcinoma cells. Cancer 117: 2926-2938. 
Dell'Antone P (2021) Energy metabolism of cancer: A suspected role of low Glutamine supply in the Warburg/Crabtree effects

49. Yamascita H, Takenoscita M, Sakurai M (2001) A glucose-responsive transcription factor that regulates carbohydrate metabolism in the liver. Proc Natl Acad Sci USA 98: 9116-9121.

50. Tong X, Zhao F, Mancuso A (2009) The glucose -responsive transcription factor ChREBP contributes to glucose-dependent anabolic synthesis and cell proliferation. Proc Nat Acad Sci USA 108: 21660-21665.
51. Wellen KE, Hatzivassiliou G, Sackdeva UM (2009) ATP-citrate lyase links cellular metabolism to histone acetylation. Science 324: 1076-1080. [Crossref]

52. Rathmell JC, Newgard CB (2009) Biochemistry. A glucose-to-gene link. Science 324: $1021-1022$.

Copyright: (C2021 Dell'Antone P. This is an open-access article distributed under the terms of the Creative Commons Attribution License, which permits unrestricted use, distribution, and reproduction in any medium, provided the original author and source are credited. 